\title{
The impact and efficacy of
} surveillance in patients with sarcoma of the extremities

\section{P. Cool, G. Cribb}

Robert Jones and Agnes Hunt Orthopaedic Hospital NHS Foundation Trust, Gobowen, Oswestry, United Kingdom
- P. Cool, MD, MMedSc(Res) DipStat FRCS(Ed) FRCS(Orth), Consultant Orthopaedic and Oncological Surgeon, - G. Cribb, MBCHB MRCS FRCS(Orth), Consultant Orthopaedic and Oncological Surgeon, Robert Jones and Agnes Hunt Orthopaedic Hospital NHS Foundation Trust, Gobowen, Oswestry SY10 7AG, UK

Correspondence should be sent to P. Cool; email paul.cool@nhs.net

doi: 10.1302/2046-3758.64.BJR2016-0253.R1

Bone Joint Res 2017;6:224-230 Received: 3 October 2016; Accepted: 6 February 2017

\section{Objectives}

In this cross sectional study, the impact and the efficacy of a surveillance programme for sarcomas of the extremities was analysed.

Methods

All patients who had treatment with curative intent for a high-grade sarcoma and were diagnosed before 2014 were included and followed for a minimum of two years.

\section{Results}

Of the 909 patients who had a review appointment in 2014, 131 were under review for a high-grade sarcoma of the extremities following treatment with curative intent. Of these patients, three patients died of disease, two patients died of other causes, 12 are alive, with disease, and 114 have no evidence of disease. The surveillance programme accounts for $14 \%$ of all review appointments.

Four of five patients $(80 \%)$ who developed local recurrence identified the recurrence themselves. Chest radiographs are adequate in identifying metastatic disease and 11 (73\%) of metastases were diagnosed during a routine follow up visit. However, the chance of cure is small and only two patients were referred for a metastatectomy. Of these only one survived for more than two years.

The mean time for developing metastatic disease and local recurrence was 2.0 and 3.9 years respectively. Once identified, the mean time to death was 2.1 years for patients with metastatic disease.

\section{Conclusions}

Surveillance of sarcoma patients makes up a substantial amount of the workload of a sarcoma unit. The chance of cure following identification of local recurrence or metastatic disease, however, is small. Alternative methods of surveillance that allow better evaluation of the patient's needs are recommended.

Cite this article: Bone Joint Res 2017;6:224-230.

Keywords: Sarcoma, Surveillance, Efficacy

\section{Article focus}

Efficacy of the surveillance programme for sarcoma

- Identification of local recurrence and metastatic disease in patients with sarcoma

\section{Key messages:}

- A substantial amount of appointments in a sarcoma unit are for surveillance following curative treatment
- Although chest radiographs are good in identifying metastatic disease, local recurrence is usually identified by the patient

- The chance of cure following the identification of local recurrence or metastatic disease is small

\section{Strengths and limitations}

- Single unit prospective cross sectional study

- Possible selection bias with limited follow up 
Introduction

Following the diagnosis and treatment of a sarcoma, patients are usually followed up to monitor the disease and outcome. The British Sarcoma Group has published standards for the surveillance of patients with sarcoma..$^{1,2}$ The standards described are in common practice in the United Kingdom. ${ }^{3}$

Imaging strategies for the identification of metastatic disease or local recurrence of sarcoma have been well described. ${ }^{4,5}$ Although chest radiographs are useful in identifying metastatic disease, unfortunately only very few patients with metastatic disease are ultimately cured. Detecting local recurrence can be more difficult and most patients identify recurrent disease themselves. Furthermore, following the identification of local recurrence, patients are seldom cured. ${ }^{5}$

In the United Kingdom, the National Institute for Health and Care Excellence (NICE) has recently updated the referral guidelines for patients with suspected sarcomas. 7,8

Most studies reviewing the efficacy of the surveillance programme are longitudinal in design; 6,9 following patients in time from diagnosis. Although useful for assessing the efficacy of the surveillance programme for individual patients; a cross sectional study design is required to analyse the impact of the surveillance programme on the provision of sarcoma services.

The aim of the present cross sectional study was firstly to investigate the impact the surveillance programme has on the provision of sarcoma services at our institution and secondly to review the efficacy of the surveillance programme.

\section{Patients and Methods}

Data were collected prospectively and stored on a database that was cross-referenced with the hospital appointments database. A cross sectional study was performed, identifying all patients who were reviewed in 2014 at the Oncology clinic of the Robert Jones and Agnes Hunt Orthopaedic Hospital NHS Foundation Trust. All patients who were under surveillance following completed treatment for a high-grade sarcoma of the extremities were identified and followed up to January 2016. The minimum follow up therefore was two years. Only patients who had treatment with curative intent were included in this study. Patients who had indeterminate lung nodules and were subsequently diagnosed with metastatic lung disease were excluded. Low-grade sarcomas were excluded because of their different mode of recurrence. Skin and trunk sarcomas were also excluded.

All patients were reviewed according to a standard protocol with review every three months following completion of treatment up to two years following diagnosis. Subsequent surveillance was every six months up to five years and yearly thereafter.
At clinical review, patients who had treatment for a soft-tissue sarcoma had a posteroanterior radiograph of the chest and clinical evaluation for local recurrence at every consultation. Patients who had treatment for a bone tumour had, in addition, plain radiographs of the affected limb. If there were any concerns on the chest radiograph, a CT scan of the chest was requested. Suspicion for local recurrence usually resulted in a MRI of the affected limb and a CT scan for patients who had a bone tumour.

Patients who had died, developed a local recurrence or metastatic disease were identified and classified accordingly. Survival analysis was performed using the Kaplan-Meier Aalen-Johansen product limit method with $95 \%$ confidence intervals. ${ }^{12}$ Statistical analysis was performed with $\mathrm{R}$ statistical software ( $\mathrm{R}$ Foundation for Statistical Computing, Vienna, Austria) ${ }^{10}$ including the survival ${ }^{11,12}$ and ggplot2 packages. ${ }^{13}$

\section{Results}

Impact of surveillance on the sarcoma service. In 2014, there were 3755 outpatient consultations for 1801 patients. Of these 1801 patients, 909 (50\%) patients had review appointments. 131 patients (14\%), of these 909, were under surveillance following completion of curative treatment for a high-grade sarcoma of the extremities (Fig. 1). These 131 patients had, in total, 360 review appointments (13\% of all review appointments). A total of 67 patients (51\%) had treatment for a high-grade sarcoma of bone and 64 patients (49\%) for a soft-tissue sarcoma. The compliance with the surveillance programme for patients who had treatment with curative intend was very high (93\%). Some patients preferred surveillance at their local hospital. There were no patients lost to follow-up.

Of the 131 patients that attended for surveillance in 2014, 40 patients were diagnosed in 2013, 22 in 2012, 13 in 2011, 19 in 2010 and 37 in or before 2009 (Fig. 2). Most patients were reviewed according to the British Sarcoma Group guidance. However, there were six patients who had more than five follow up consultations in 2014 (Fig. 3). Of these, three patients had no evidence of recurrent disease. Two of these patients (seven and eight reviews) had mechanical problems relating to the reconstruction of a bone tumour and one patient (14 reviews) had multiple appointments for an infected seroma following treatment with surgery and radiotherapy for a soft-tissue sarcoma of the thigh.

The remaining three patients had follow up consultations related to recurrent disease. One patient (six reviews) for the diagnosis of a local recurrence, one patient (seven reviews) had concerns she had local recurrence, but this turned out not to be the case. The final patient, who was reviewed 11 times, initially had successful two-stage revision for an infected endoprosthetic 

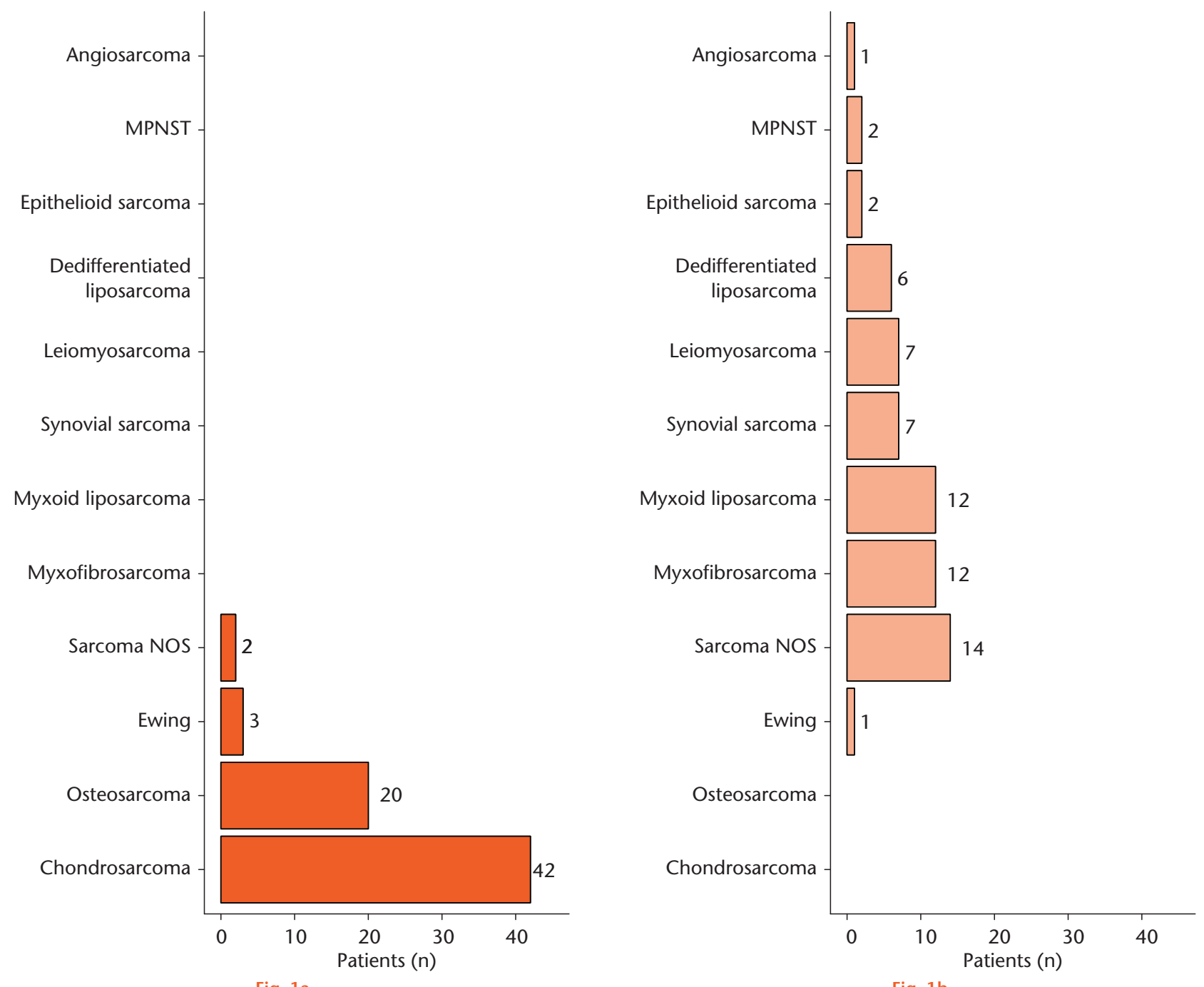

Fig. 1a

Fig. 1b

Graph showing diagnoses of 131 surveillance patients with a) high-grade bone and b) soft-tissue sarcomas (MPNST, malignant peripheral nerve sheath tumour; NOS, not otherwise specified).

replacement of the proximal tibia but subsequently developed metastatic disease.

Efficacy of the surveillance programme. Of the 131 patients, three patients (2\%) died of disease, two died of another cause (2\%), 12 are alive with disease (9\%) and 114 patients have no evidence of disease (87\%) (Fig. 4). There was no significant difference in survival between males and females or between bone and soft-tissue tumours.

Local recurrence. The local recurrence free survival is shown in Figure 5. In total, five patients developed local recurrence (four soft-tissue sarcomas and one bone sarcoma). All but one patient (80\%) noticed the local recurrence himself or herself. The other patient had the local recurrence identified during a routine surveillance visit. The mean time from diagnosis to local recurrence was 3.9 years.

One patient died of disease, two patients are alive with disease and two patients have no further evidence of disease, to date.
Metastases. Overall, 15 patients developed metastatic disease (Fig. 6), 11 (73\%) of the patients with metastatic disease had this identified by the surveillance programme's chest radiographs. The other four patients (27\%) had symptoms and requested interim review. However, only two patients (13\%) were subsequently referred for metastatectomy of which one died and one is alive with disease.

The mean time for patients to develop metastatic disease was two years following diagnosis with a mean further survival of 2.1 years.

A total of 10 of these patients are alive with disease, two died of disease and three have no further evidence of disease to date. Nine of the 15 patients were under surveillance following treatment for a bone sarcoma (five osteosarcomas, two chondrosarcomas and two Ewing's sarcomas) and six had treatment for a soft-tissue sarcoma.

Survival. Three patients died of disease (two soft-tissue sarcomas and one a bone sarcoma). The Kaplan-Meier 


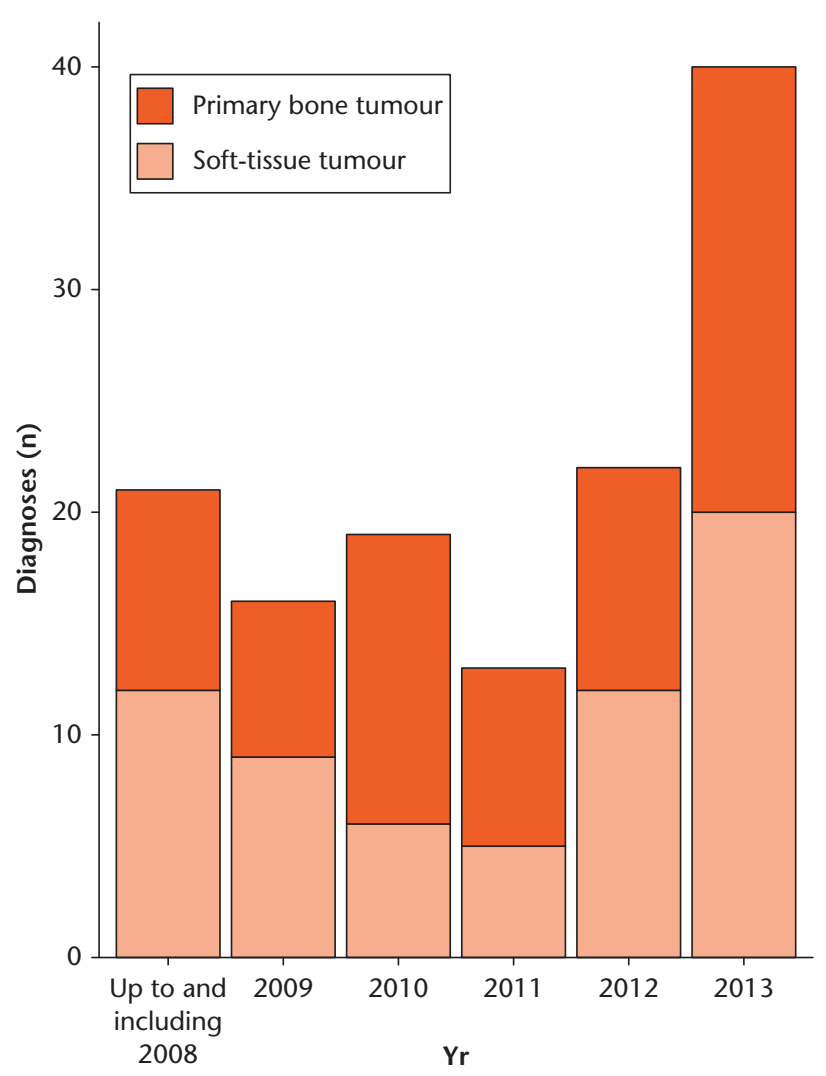

Fig. 2

Graph showing the number of newly diagnosed high-grade bone and softtissue sarcomas grouped by year of diagnosis.

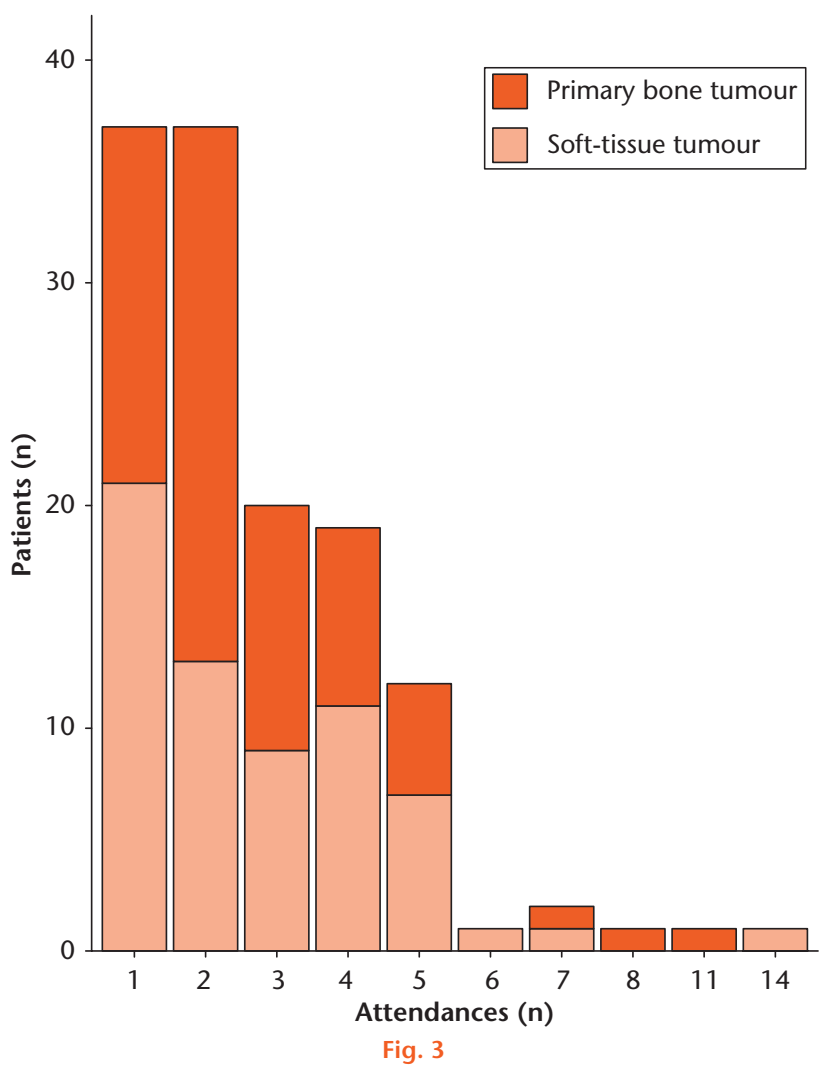

Graph showing the number of reviews for 131 patients under surveillance with high-grade sarcoma of the extremities.

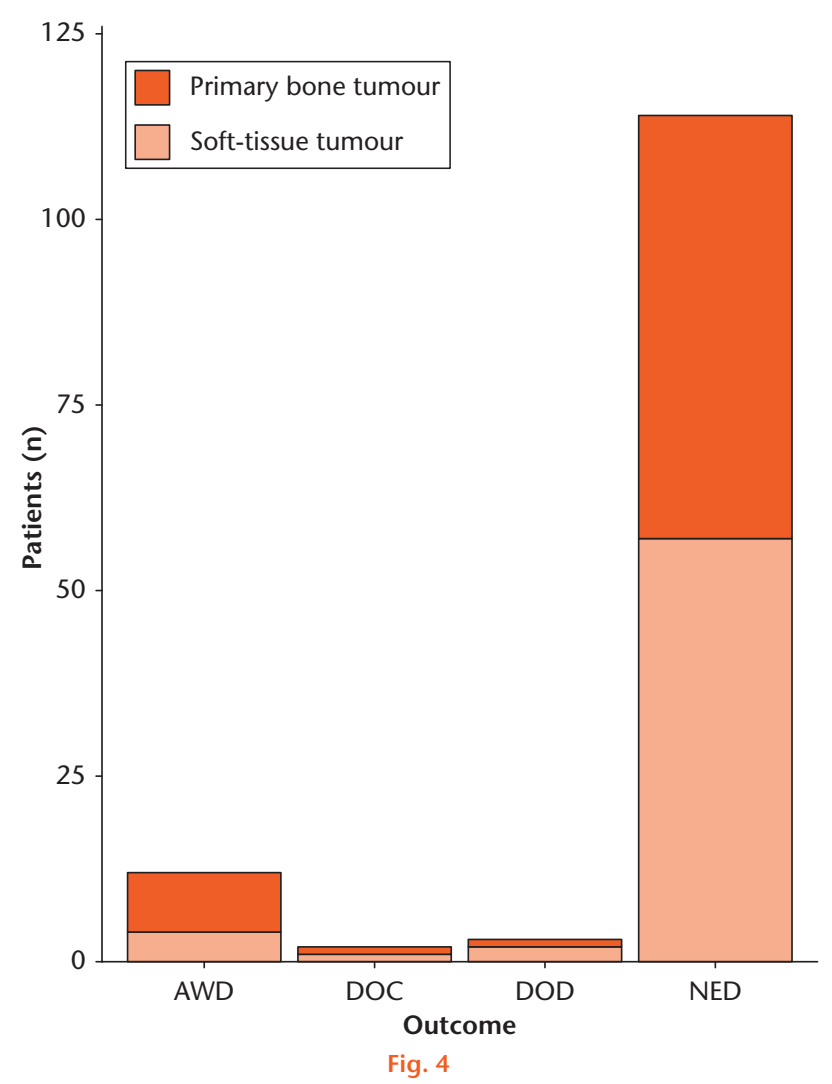

Graph showing the oncological outcome of 131 patients with high-grade sarcoma of the extremities. (AWD, alive with disease; DOC, died of other cause; DOD, died of disease; NED, no evidence of disease).

overall survival curve is depicted in Figure 7. One patient with Ewing's sarcoma developed metastatic disease that was identified on a chest radiograph during a routine surveillance visit 1.5 years following diagnosis. This patient lived for a further ten months. Another patient presented 2.2 years following diagnosis with pain suggestive of local recurrence of a pleomorphic sarcoma of soft tissue. Investigations confirmed local recurrence as well as metastatic disease. The patient survived a further 2.8 years following palliative treatment. The third patient was under surveillance following treatment for a synovial sarcoma of the upper arm that was treated with excision and radiotherapy. Two years following treatment the patient developed lung metastases that were successfully resected. A subsequent radiation associated fracture was treated successfully, but the patient developed lymph node disease 4.5 years later, and subsequently died of disease.

\section{Discussion}

This study showed that $14 \%$ of the follow up appointments at our institution are for the surveillance of patients who had treatment for a high-grade sarcoma of the extremities. Chest radiographs identified $73 \%$ of metastases, but only $13 \%$ of all patients who developed metastatic disease were referred for metastatectomy. Although these patients had a prolonged disease free interval, none 


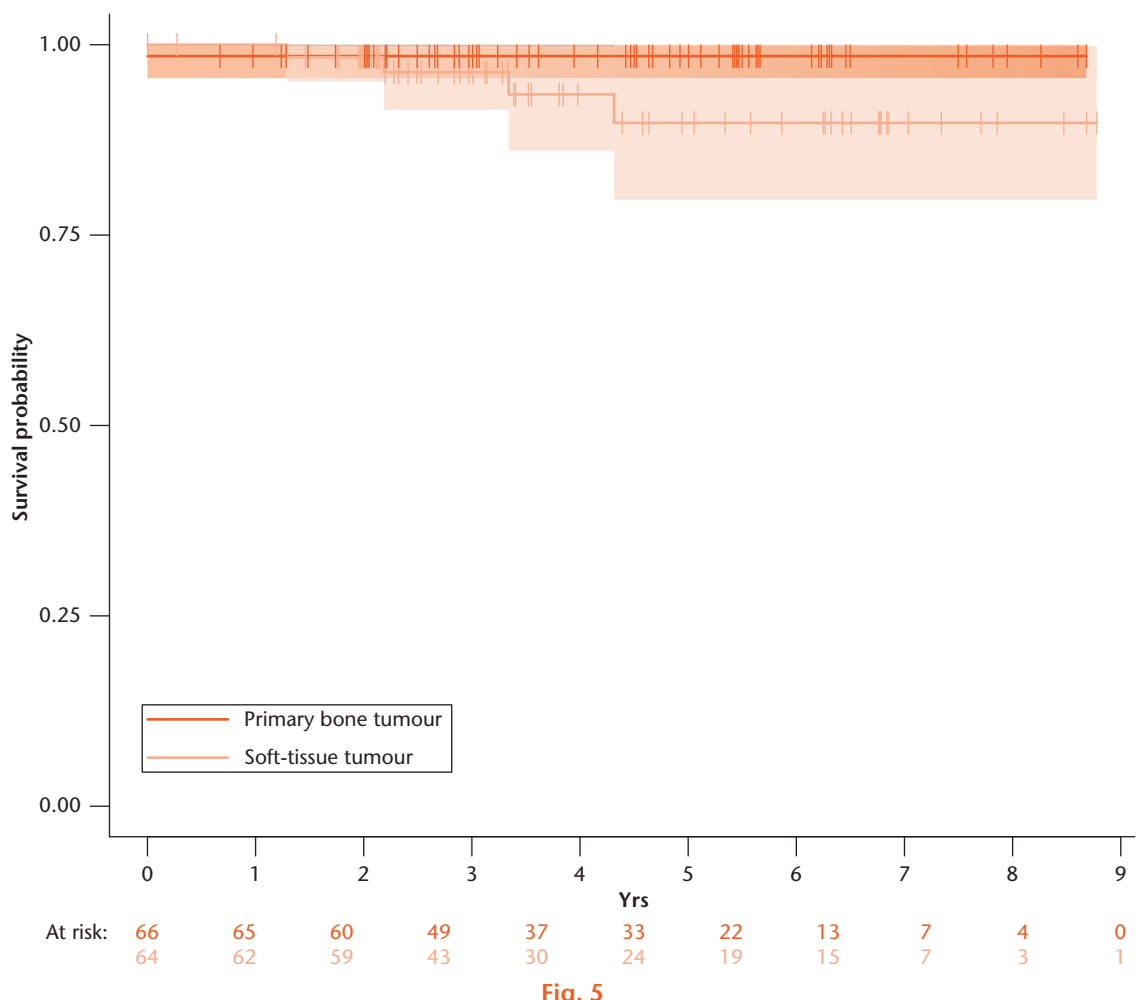

Graph showing local recurrence free Kaplan-Meier Aalen-Johansen survival of 131 patients under surveillance with high-grade sarcoma of the extremities Log-rank: $p=0.1$.

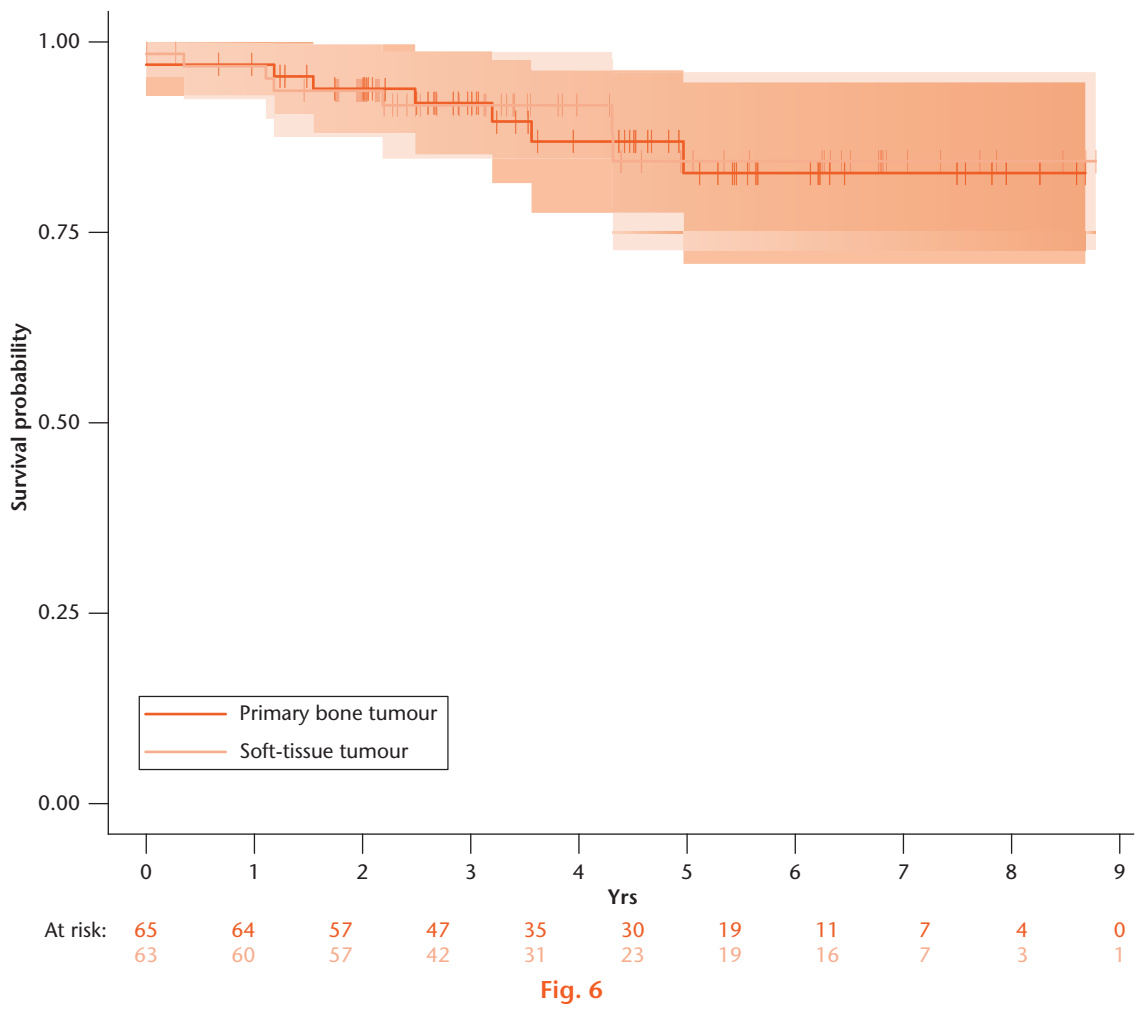

Graph showing the metastatic free Kaplan-Meier Aalen-Johansen survival of 131 patients under surveillance with high-grade sarcoma of the extremities Log-rank: $p=0.9$. 


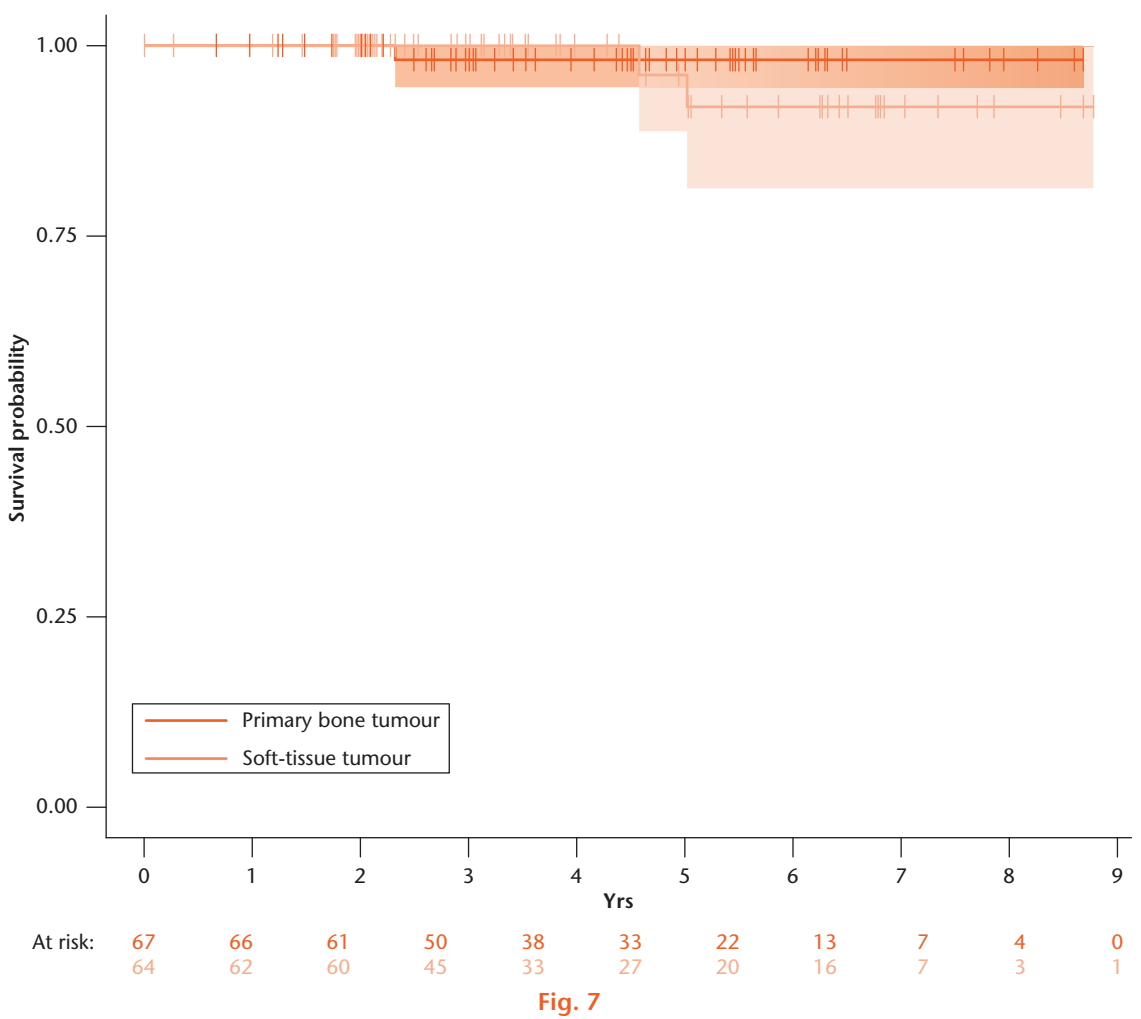

Graph showing the overall Kaplan-Meier Aalen-Johansen survival of 131 patients under surveillance with high-grade sarcoma of the extremities. Log-rank: $\mathrm{p}=0.5$.

of these patients were ultimately cured of their disease. As reported previously ${ }^{6}$ the vast majority ( $80 \%$ ) of patients identify local recurrent disease themselves. Puri et $\mathrm{al}^{14}$ also showed in a prospective trial that inexpensive imaging combined with patient education regarding selfexamination will detect most local recurrences. Based on these data, an alternative approach for surveillance seems justified.

Goel et $\mathrm{al}^{15}$ tried to quantify the cost of the surveillance regime in a meta-analysis of 34 articles and identified 54 different strategies. Clinical surveillance and chest radiographs were the most commonly used methods. They concluded that a randomised controlled trial is required. However, such a trial is difficult to set up. Kane et $\mathrm{al}^{16}$ suggested that clinical history, patient education and thorough clinical examination identified the majority of recurrent disease. They concluded that image surveillance of the chest is only indicated in high-risk cases, or if clinical evaluation is difficult. Similarly, Lord et al ${ }^{17}$ suggested that chest radiographs are indicated in the surveillance of high-grade tumours, but not low-grade tumours. Christie-Large et al ${ }^{18}$ suggested that chest radiographs followed by CT on indication detects $93 \%$ of chest metastases. Whooley et al ${ }^{19}$ agree that chest radiographs are the most valuable imaging modality for the detection of metastatic disease in the chest.

Long term results following pulmonary metastectomy are encouraging, particularly if there is a long disease free interval and the resection margins are clear. ${ }^{20,21}$ Pfannschmidt et al and Rehders et a|22,23 agree that metastectomy can be useful and increase survival with a low complication rate. However, ultimate cure is rare and patients are less likely to benefit from pulmonary metastatectomy if they also have locally recurrent disease. ${ }^{24}$

Much less is published regarding the surveillance of bone sarcomas. ${ }^{6}$ However, it has been suggested that, in addition, bone scintigraphy can be useful in the follow up of osteosarcoma. 25

MRI and ultrasound scan can both be useful in surveillance to detect local recurrence. In particular, ultrasound scans as an initial screening tool with MRI for inconclusive cases. ${ }^{26}$ However, it is not our normal practice to routinely perform MRI scans for surveillance. Positron Emission Computerised Tomography (PET CT) may be useful as an adjuvant, but its roles remain largely undetermined. Ultrasound scans can also be useful to guide biopsies of suspected local recurrences. ${ }^{26}$

Tseng et al ${ }^{27}$ advocate a more personalised approach to surveillance and Nakamura et al ${ }^{28}$ have described a scale to predict patient survival. A clinical review is unlikely to address all patients concerns and perhaps a more holistic approach in surveillance is justified. This could include the introduction of patient support groups and additional psychological/nursing support. As most patients identify local recurrent disease themselves, 
self-surveillance with instructions on how to identify local recurrence seems justified. Plain radiographs of the chest could be performed regularly and reported the same day to reduce patient anxiety regarding the result.

Although a cross sectional study evaluates the impact of the surveillance programme on the clinical service better, it also has limitations. Its design makes selection bias more likely and the follow up is relatively short. However, most recurrent disease in high-grade tumours is detected within this time frame. Furthermore, many patients have multimodal treatment and have additional surveillance by their medical/radiation oncologist as appropriate. Therefore, the impact on resources of the surveillance programme is likely to be higher. Our clinical practice mainly involves adults, explaining the relatively high incidence of chondrosarcomas; which are more frequent in the adult population.

Despite a significant use of resources, the overall efficacy of the surveillance programme is low. Furthermore, it is unlikely that all patient's concerns are addressed during a relatively short follow-up consultation and perhaps alternative methods of surveillance that better address individual patient's needs need to be explored. A holistic approach with assessment by the nurses and physiotherapist combined with patient support groups and educational sessions may be more appropriate and useful for the patient.

\section{References}

1. Grimer R, Judson I, Peake D, Seddon B. Guidelines for the Management of Soft Tissue Sarcomas. Sarcoma 2010:1-15.

2. Grimer R, Athanasou N, Gerrand C, et al. UK Guidelines for the Management of Bone Sarcomas. Sarcoma 2010:1-14.

3. Gerrand CH, Billingham LJ, Woll PJ, Grimer RJ. Follow up after Primary Treatment of Soft Tissue Sarcoma: A Survey of Current Practice in the United Kingdom. Sarcoma 2007:1-6.

4. Bearcroft PW, Davies AM. Follow-up of musculoskeletal tumours. 2. Metastatic disease. Eur Radiol 1999;9:192-200.

5. Davies AM, Vanel D. Follow-up of musculoskeletal tumors. I. Local recurrence. Eur Radiol 1998;8:791-799.

6. Cool P, Grimer R, Rees R. Surveillance in patients with sarcoma of the extremities. Eur J Surg Oncol 2005;31:1020-1024.

7. National Institute for Clinical Excellence (Great Britain). Improving outcomes for people with sarcoma: the manual. London: National Institute for Clinical Excellence; 2006.

8. No authors listed. National Insitute for Health and Care Excellence. Sarcoma Guidance and guidelines. https://www.nice.org.uk/guidance/qs78 (date last accessed 23 February 2017)

9. Rothermundt C, Whelan JS, Dileo P, et al. What is the role of routine follow-up for localised limb soft tissue sarcomas? A retrospective analysis of 174 patients. Br J Cancer 2014;110:2420-2426.
10. No authors Listed. $R$ Foundation for Statistical Computing $A$ language and environment for statistical computing https://www.R-project.org/ (date last accessed 23 February 2017)

11. Therneau T. Survival: a package for survival analysis in R. R package version 2.37. 2014.

12. Gerds TA. prodlim: Product-limit estimation for censored event history analysis 2014 http://CRAN.R-project.org/package=prodlim (date last accessed 23 February 2017)

13. Wickham H. ggplot2: elegant graphics for data analysis. New York: Springer Verlag; 2009. http://cran.r-project.org/package=ggplot2 (date last accessed 23 February 2017)

14. Puri A, Gulia A, Hawaldar R, et al. Does intensity of surveillance affect survival after surgery for sarcomas? Results of a randomized noninferiority trial. Clin Orthop Relat Res 2014;472:1568-1575.

15. Goel A, Christy MEL, Virgo KS, et al. Costs of follow-up after potentially curative treatment for extremity soft-tissue sarcoma. Int J Oncol 2004;25:429-435.

16. Kane JM III. Surveillance strategies for patients following surgical resection of soft tissue sarcomas. Curr Opin Oncol 2004;16:328-332.

17. Lord HK, Salter DM, MacDougall RH, Kerr GR. Is routine chest radiography a useful test in the follow up of all adult patients with soft tissue sarcoma? Br J Radiol 2006;79:799-800.

18. Christie-Large M, James SLJ, Tiessen L, et al. Imaging strategy for detecting lung metastases at presentation in patients with soft tissue sarcomas. Eur J Cancer, 44:1841-1845.

19. Whooley BP, Gibbs JF, Mooney MM, et al. Primary extremity sarcoma: what is the appropriate follow-up? Ann Surg Onco/ 2000;7:9-14.

20. García Franco CE, Torre W, Tamura A, et al. Long-term results after resection for bone sarcoma pulmonary metastases. Eur J Cardiothorac Surg 2010;37:1205-1208.

21. Smith R, Pak Y, Kraybill W, Kane JM III. Factors associated with actual long-term survival following soft tissue sarcoma pulmonary metastasectomy. Eur J Surg Oncol 2009;35:356-361.

22. Pfannschmidt J, Klode J, Muley T, et al. Pulmonary metastasectomy in patients with soft tissue sarcomas: experiences in 50 patients. Thorac Cardiovasc Surg 2006;54:489-492.

23. Rehders A, Hosch SB, Scheunemann P, et al. Benefit of surgical treatment of lung metastasis in soft tissue sarcoma. Arch Surg 2007;142:70-75.

24. Chen F, Fujinaga T, Sato K, et al. Significance of tumor recurrence before pulmonary metastasis in pulmonary metastasectomy for soft tissue sarcoma. Eur J Surg Oncol 2009;35:660-665.

25. Körholz D, Wirtz I, Vosberg H, et al. The role of bone scintigraphy in the follow-up of osteogenic sarcoma. Eur J Cancer 1996;32A:461-464.

26. Choi H, Varma DG, Fornage BD, et al. Soft-tissue sarcoma: MR imaging vs sonography for detection of local recurrence after surgery. AJR Am J Roentgenol 1991;157:353-358.

27. Tseng WW, Amini B, Madewell JE. Follow-up of the soft tissue sarcoma patient. J Surg Oncol 2015;111:641-645.

28. Nakamura T, Matsumine A, Asanuma K, et al. The value of the high-sensitivity modified Glasgow prognostic score in predicting the survival of patients with a softtissue sarcoma. Bone Joint J [Br]2015;97-B:847-852.

Funding Statement

- This research received no specific grant from any funding agency in the public, commercial or not-for-profit sectors.

Author Contribution

- P. Cool: Design, collection of data, analysis, writing paper

G. Cribb: Design, collection of data, writing paper, review.

ICMJE COI Statement

- None declared

(c) 2017 Cool et al. This is an open-access article distributed under the terms of the Creative Commons Attributions licence (CC-BY-NC), which permits unrestricted use, distribution, and reproduction in any medium, but not for commercial gain, provided the original author and source are credited. 